\title{
Diffusion coefficient fractal dimension for characterizing Shajara reservoirs of the Permo - carboniferous Shajara formation, Saudi Arabia
}

\begin{abstract}
The quality and assessment of a reservoir can be documented in details by the application of diffusion coefficient. This research aims to calculate fractal dimension from the relationship among diffusion coefficient, maximum diffusion coefficient and wetting phase saturation and to approve it by the fractal dimension derived from the relationship among capillary pressure and wetting phase saturation. In this research, porosity was measured on real collected sandstone samples and permeability was calculated theoretically from capillary pressure profile measured by mercury intrusion contaminating the pores of sandstone samples in consideration. Two equations for calculating the fractal dimensions have been employed. The first one describes the functional relationship between wetting phase saturation, diffusion coefficient, maximum diffusion coefficient and fractal dimension. The second equation implies to the wetting phase saturation as a function of capillary pressure and the fractal dimension. Two procedures for obtaining the fractal dimension have been utilized. The first procedure was done by plotting the logarithm of the ratio between diffusion coefficient and maximum diffusion coefficient versus logarithm wetting phase saturation. The slope of the first procedure $=3$ - Df (fractal dimension). The second procedure for obtaining the fractal dimension was determined by plotting the logarithm of capillary pressure versus the logarithm of wetting phase saturation. The slope of the second procedure $=\mathrm{Df}-3$. On the basis of the obtained results of the fabricated stratigraphic column and the attained values of the fractal dimension, the sandstones of the Shajara reservoirs of the Shajara Formation were divided here into three units. The obtained units from bottom to top are: Lower, Middle and Upper Shajara Diffusion Coefficient Fractal Dimension Units. It was found that fractal dimension increases with increasing grain size and permeability.
\end{abstract}

Keywords: Shajara reservoirs, Shajara formation, diffusion coefficient fractal dimension, capillary pressure fractal dimension
Volume 4 Issue 2 - 2019

\author{
Khalid Elyas Mohamed Elameen Alkhidir \\ Department of Petroleum and Natural Gas Engineering, College \\ of Engineering, King Saud University, Saudi Arabia
}

\begin{abstract}
Correspondence: Prof. Khalid Elyas Mohamed Elameen Alkhidir, Ph.D, Department of Petroleum and Natural Gas Engineering, College of Engineering, King Saud University, Riyadh,
\end{abstract} Saudi Arabia,Tel +966 | |4679 | 18, Email kalkhidir@ksu.edu.sa

Received: March 21, 2019 | Published: April 05, 2019

\section{Introduction}

Seismo electric effects related to electro kinetic potential, dielectric permittivity, pressure gradient, fluid viscosity, and electric conductivity was first reported. ${ }^{1}$ Capillary pressure follows the scaling law at low wetting phase saturation was reported. ${ }^{2}$ Seismo electric phenomenon by considering electro kinetic coupling coefficient as a function of effective charge density, permeability, fluid viscosity and electric conductivity was reported. ${ }^{3}$ The magnitude of seismo electric current depends porosity, pore size, zeta potential of the pore surfaces, and elastic properties of the matrix was investigated. ${ }^{4}$ The tangent of the ratio of converted electric field to pressure is approximately in inverse proportion to permeability was studied. Permeability inversion from seism electric log at low frequency was studied. ${ }^{6}$ They reported that, the tangent of the ratio among electric excitation intensity and pressure field is a function of porosity, fluid viscosity, frequency, tortuosity, fluid density and Dracy permeability. A decrease of seismo electric frequencies with increasing water content was reported. ${ }^{7}$ An increase of seismo electric transfer function with increasing water saturation was studied. ${ }^{8}$ An increase of dynamic seismo electric transfer function with decreasing fluid conductivity was described. ${ }^{9}$ The amplitude of seismo electric signal increases with increasing permeability which means that the seismo electric effects are directly related to the permeability and can be used to study the permeability of the reservoir was illustrated. ${ }^{10}$ Seismo electric coupling is frequency dependent and decreases expontialy when frequency increases was demonstrated. ${ }^{11}$ An increase of permeability with increasing pressure head and bubble pressure fractal dimension was reported. ${ }^{12}$ An increase of geometric and arithmetic relaxation time of induced polarization fractal dimension with permeability increasing was described. ${ }^{13-15}$

\section{Material and method}

Sandstone samples were collected from the surface type section of the Permo-Carboniferous Shajara Formation, latitude $26^{\circ} 52^{\prime} 17.4$ ', longitude $43^{\circ} 36^{\prime} 18^{\prime \prime}$ (Figure 1). Porosity was measured on collected samples using mercury intrusion Porosimetry and permeability was derived from capillary pressure data. The purpose of this paper is to obtain diffusion coefficient fractal dimension and to confirm it by capillary pressure fractal dimension. The fractal dimension of the first procedure is determined from the positive slope of the plot of logarithm of the ratio of diffusion coefficient to maximum diffusion coefficient $\log \left(\mathrm{D}^{1 / 2} / \mathrm{D}_{\max }^{1 / 2}\right)$ versus $\log$ wetting phase saturation $(\log \mathrm{Sw})$. Whereas the fractal dimension of the second procedure is determined from the negative slope of the plot of logarithm of $\log$ capillary pressure $(\log \mathrm{Pc})$ versus logarithm of wetting phase saturation $(\log \mathrm{Sw})$. 


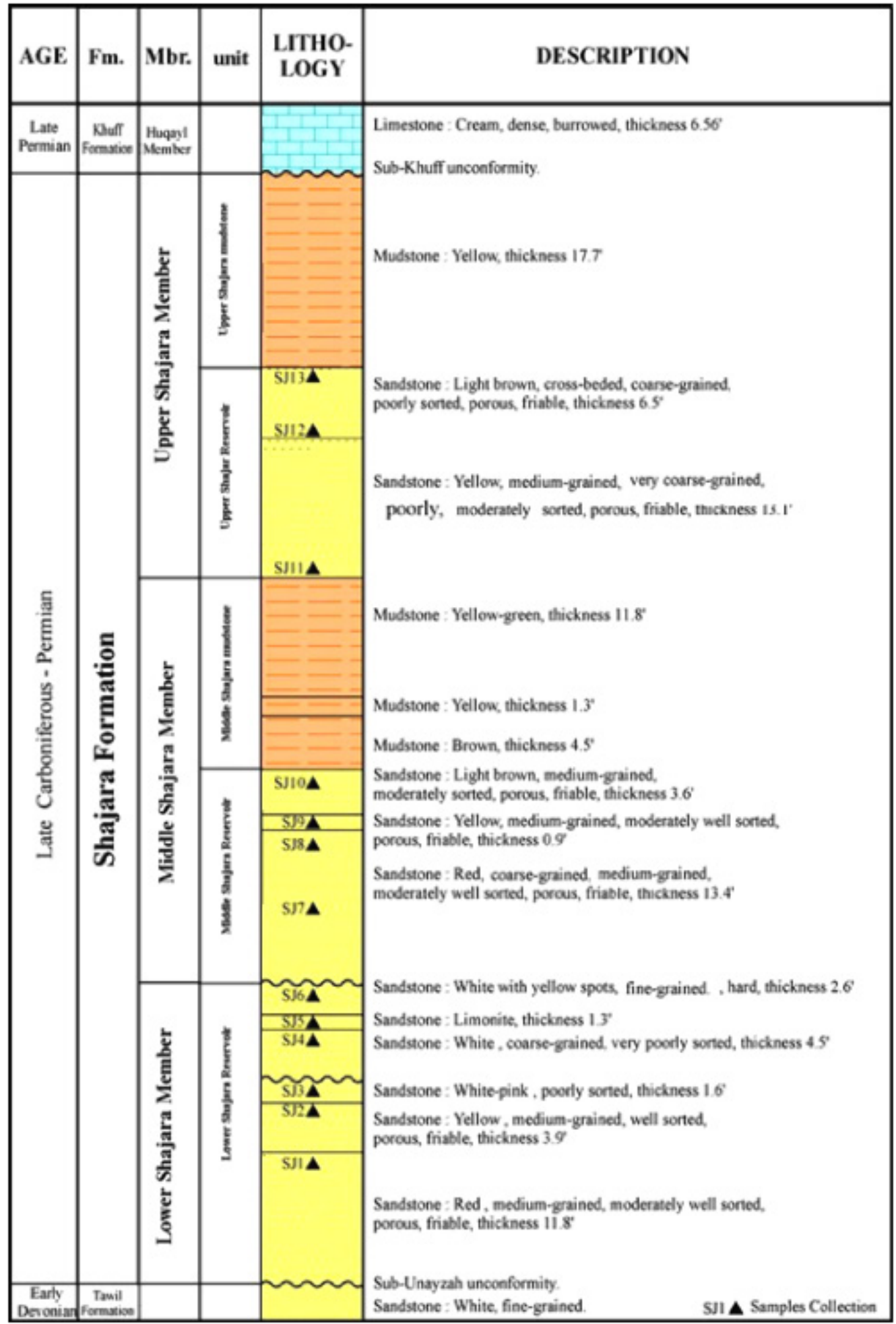

Figure I Surface type section of the Shajara Reservoirs of the Permo - Carboniferous Shajara Formation at latitude $26^{\circ} 52^{\prime} 17.4^{\prime \prime}$, longitude $43^{\circ} 36^{\prime} 18^{\prime \prime}$.

The diffusion coefficient can be scaled as

$$
S w=\left[\frac{D^{\frac{1}{2}}}{D \max ^{\frac{1}{2}}}\right]^{3-D f}
$$

Equation 1 can be proofed from

$$
D e=\frac{\left[D^{*} \phi^{*} \text { connectivity }\right]}{T}
$$

Where $D e$ the effective diffusion coefficient in square micrometer per second; D diffusion coefficient in square micrometer per second; 
$\phi$ porosity (dimensionless); connectivity (dimensionless); and $\mathrm{T}$ tortuosity (dimensionless).

The diffusion coefficient can be scaled as

$$
D=\frac{A^{2}}{[2 * T c c]}
$$

Where $\mathrm{D}$ the diffusion coefficient in square micrometer per second; A pore throat radius in micrometer; Tcc cole time in seconds. The effective diffusion coefficient can be scaled as

$$
D e=\frac{A e^{2}}{[2 * \text { Tcceff }]}
$$

Where $D e$ the effective diffusion coefficient in square micrometer per second; Ae effective pore throat radius in micrometer; and Tcceff the effective cole time in seconds. Insert equation 4 into equation 2.

$$
\frac{A e^{2}}{[2 * T c c e f f]}=\frac{\left[D^{*} \phi^{*} \text { connectivity }\right]}{T}
$$

The effective pore throat radius can be scaled as

$$
A e^{2}=8 * k * F
$$

Where $A e$ the effective pore throat radius in micrometer; $\mathrm{k}$ permeability in square micrometer; and $\mathrm{F}$ the formation resistivity factor (dimensionless). Insert equation 6 into equation 5

$$
\frac{[8 * k * F]}{[2 * T c c e f f]}=\frac{[D * \phi * \text { connectivity }]}{T}
$$

Equation 7 after simplification and division both sides by $\phi$ will become

$$
\frac{\left[4 * k^{*} F\right]}{[\text { Tcceff } * \phi]}=\frac{\left[D * \phi^{*} \text { connectivity }\right]}{T * \phi}
$$

Equation 8 after simplification will become

$$
\frac{[4 * k * F]}{[\text { Tcceff } * \phi]}=\frac{[D * \text { connectivity }]}{T}
$$

Equation 9 after rearrange will become

$$
\frac{k}{\phi}=\frac{\left[D^{*} \text { connectivity } * \text { Tcceff }\right]}{\left[4 * k^{*} F\right]}
$$

Take the square root of equation 10

$$
\begin{aligned}
& \sqrt{\frac{k}{\phi}}=\sqrt{\frac{\left[D^{*} \text { connectivity } * \text { Tcceff }\right]}{\left[4 * k^{*} F\right]}} \\
& \text { But; } \sqrt{\frac{k}{\phi}}=R Q I
\end{aligned}
$$

Equation 12 will become

$$
R Q I=\sqrt{\frac{[D * \text { connectivity } * \text { Tcceff }]}{[4 * k * F]}}
$$

Where RQI the reservoir quality index in micrometer. The maximum reservoir quality index can be scaled as

$$
R Q \operatorname{Im} a x=\sqrt{\frac{[D \max * \text { connectivity } * \text { Tcceff }]}{\left[4 * k^{*} F\right]}}
$$

The reservoir quality index can be scaled as

$$
S w=\left[\frac{R Q \mathrm{I}}{R Q \operatorname{Im} a x}\right]^{3-D f}
$$

Where $S w$ the water saturation; RQI reservoir quality index in micrometer; RQImax maximum reservoir quality index in micrometer; and $D f$ fractal dimension (dimensionless). Insert equation 13 into equation 15

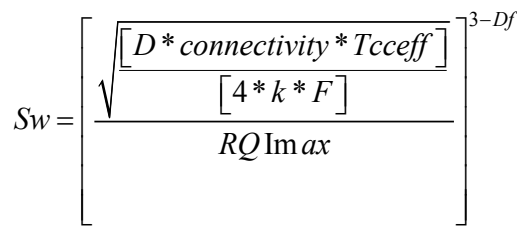

Insert equation 14 into equation 16

$$
S w=\left[\frac{\sqrt{\frac{[D * \text { connectivity*Tcceff }]}{[4 * k * F]}}}{\sqrt{\frac{[\text { Dmax*connectivity } * \text { Tcceff }]}{[4 * k * F]}}}\right]^{3-D f}
$$

Equation 17 after simplification will become

$$
S w=\left[\frac{\sqrt{D}}{\sqrt{D \max }}\right]^{3-D}=\left[\frac{D^{\frac{1}{2}}}{D \max ^{\frac{1}{2}}}\right]^{3-D f}
$$

Equation 18 is the proof of equation 1 which relates water saturation; diffusion coefficient; maximum diffusion coefficient; and fractal dimension. The capillary pressure can be scaled as

$$
\log S w=[D f-3] * \log P c+\text { constant }
$$

Where $S w$ the water saturation, Pc the capillary pressure, and $D f$ the fractal dimension

\section{Result and discussion}

Based on field observation the Shajara Reservoirs of the Shajara Formation of the Permo-Carboniferous Unayzah Group were divided here into three units as described in Figure 1. These units from bottom to top are: Lower Shajara Reservoir, Middle Shajara reservoir, and Upper Shajara Reservoir.

Their acquired results of the diffusion coefficient fractal dimension and capillary pressure fractal dimension are displayed in Table 1. Based on the attained results it was found that the diffusion coefficient fractal dimension is equal to the capillary pressure fractal dimension. The maximum value of the fractal dimension was found to be 2.7872 assigned to sample SJ13 from the Upper Shajara Reservoir as verified in Table 1. Whereas the minimum value of the fractal dimension 2.4379 was reported from sample SJ3 from the Lower Shajara reservoir as displayed in Table1. The diffusion coefficient fractal dimension and capillary pressure fractal dimension were observed to increase with increasing permeability as proofed in Table1 owing to the possibility of having interconnected channels.

The Lower Shajara reservoir was denoted by six sandstone samples (Figure 1), four of which label as SJ1, SJ2, SJ3 and SJ4 were selected 
for capillary pressure measurement as confirmed in Table 1. Their positive slopes of the first procedure (log of the ratio of diffusion coefficient to maximum diffusion coefficient versus log wetting phase saturation) and negative slopes of the second procedure (log capillary pressure versus $\log$ wetting phase saturation $(\log \mathrm{Sw})$ is delineated in Figures 2-5. Their diffusion coefficient fractal dimension and capillary pressure fractal dimension values are shown in Table 1.
As we proceed from sample SJ2 to SJ3 a pronounced reduction in permeability due to compaction was reported from $1955 \mathrm{md}$ to $56 \mathrm{md}$ which reflects decrease in diffusion coefficient fractal dimension from 2.7748 to 2.4379 as specified in Table 1. Again, an increase in grain size and permeability was verified from sample SJ4 whose diffusion coefficient fractal dimension and capillary pressure fractal dimension was found to be 2.6843 as described in Table 1 .

Table I Petrophysical model showing the three Shajara Reservoir Units with their corresponding values of diffusion coefficient fractal dimension and capillary pressure fractal dimension

\begin{tabular}{|c|c|c|c|c|c|c|c|c|}
\hline Formation & Reservoir & Sample & Porosity \% & k md & $\begin{array}{l}\text { Positive slope of } \\
\text { the first procedure } \\
\text { Slope=3-Df }\end{array}$ & $\begin{array}{l}\text { Negative slope of the } \\
\text { second procedure } \\
\text { Slope=Df-3 }\end{array}$ & $\begin{array}{l}\text { Diffusion } \\
\text { coefficient } \\
\text { fractal } \\
\text { dimension }\end{array}$ & $\begin{array}{l}\text { Capillary } \\
\text { pressure } \\
\text { fractal } \\
\text { dimension }\end{array}$ \\
\hline \multirow{10}{*}{$\begin{array}{l}\text { Permo- } \\
\text { Carboniferous } \\
\text { Shajara } \\
\text { Formation }\end{array}$} & \multirow{3}{*}{$\begin{array}{l}\text { Upper } \\
\text { Shajara } \\
\text { Reservoir }\end{array}$} & SJI3 & 25 & 973 & 0.2128 & -0.2128 & 2.7872 & 2.7872 \\
\hline & & SJI2 & 28 & 1440 & 0.2141 & $-0.2|4|$ & 2.7859 & 2.7859 \\
\hline & & SJII & 36 & 1197 & $0.24 I 4$ & $-0.24 \mid 4$ & 2.7586 & 2.7586 \\
\hline & \multirow{3}{*}{$\begin{array}{l}\text { Middle } \\
\text { Shajara } \\
\text { Reservoir }\end{array}$} & SJ9 & 31 & 1394 & 0.2214 & -0.2214 & 2.7786 & 2.7786 \\
\hline & & SJ8 & 32 & 1344 & 0.2248 & -0.2248 & 2.7752 & 2.7752 \\
\hline & & SJ7 & 35 & 1472 & 0.2317 & -0.2317 & 2.7683 & 2.7683 \\
\hline & \multirow{4}{*}{$\begin{array}{l}\text { Lower } \\
\text { Shajara } \\
\text { Reservoir }\end{array}$} & SJ4 & 30 & 176 & 0.3157 & -0.3157 & 2.6843 & 2.6843 \\
\hline & & SJ3 & 34 & 56 & 0.5621 & -0.5621 & 2.4379 & 2.4379 \\
\hline & & SJ2 & 35 & 1955 & 0.2252 & -0.2252 & 2.7748 & 2.7748 \\
\hline & & SJI & 29 & 1680 & $0.214 \mid$ & $-0.2|4|$ & 2.7859 & 2.7859 \\
\hline
\end{tabular}

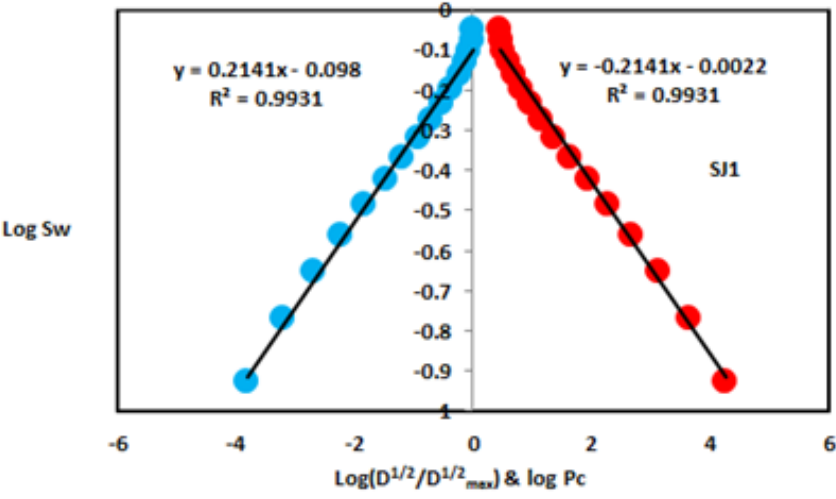

Figure $2 \log \left(D^{1 / 2} / D_{\max }{ }^{1 / 2}\right)$ \& log Pc versus log Sw for sample SJI.

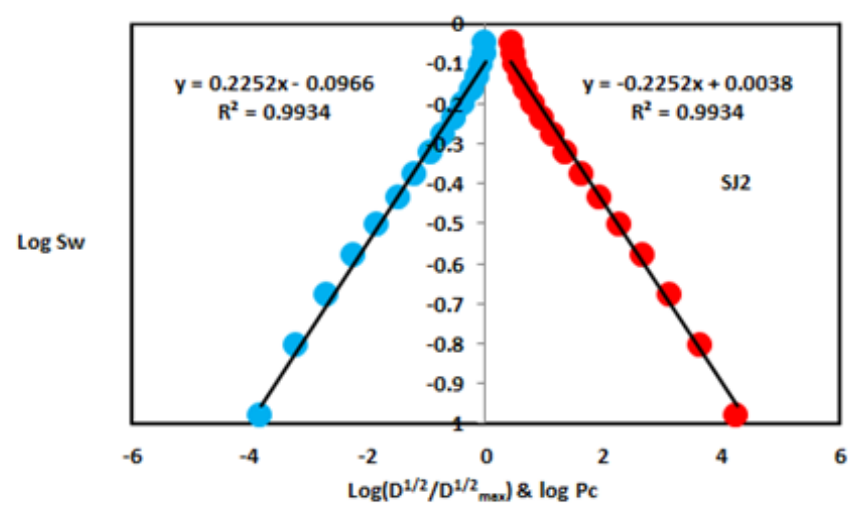

Figure $3 \log \left(D^{1 / 2} / D_{\max }{ }^{1 / 2}\right)$ \& log Pc versus log Sw for sample SJ2.

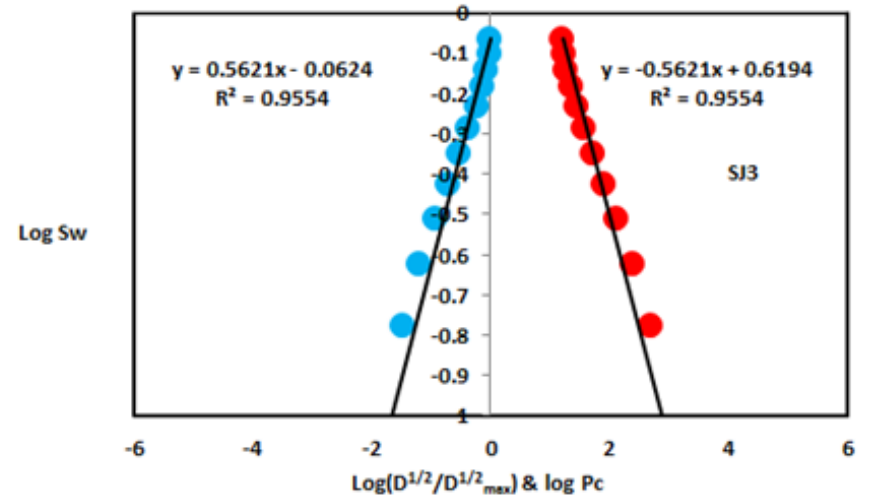

Figure 4 Log $\left(D^{1 / 2} / D_{\max }^{1 / 2}\right) \& \log P c$ versus log Sw for sample SJ3.

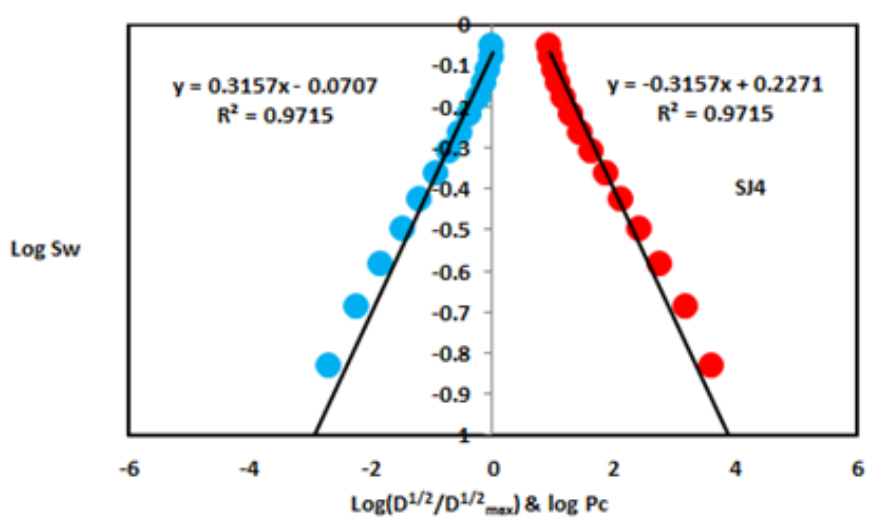

Figure 5 Log $\left(D^{1 / 2} / D_{\max }^{1 / 2}\right) \& \log P c$ versus log Sw for sample SJ4. 
In contrast, the Middle Shajara reservoir which is separated from the Lower Shajara reservoir by an unconformity surface as shown in Figure 1. It was designated by four samples (Figure 1), three of which named as SJ7, SJ8, and SJ9 as illustrated in Table 1 were selected for capillary measurements as described in Table 1. Their positive slopes of the first procedure and negative slopes of the second procedure are shown in Figures 6-8. Additionally, their diffusion coefficient fractal dimensions and capillary pressure fractal dimensions show similarities as delineated in Table 1.Their fractal dimensions are higher than those of samples SJ3 and SJ4 from the Lower Shajara Reservoir due to an increase in their permeability as explained in Table 1.

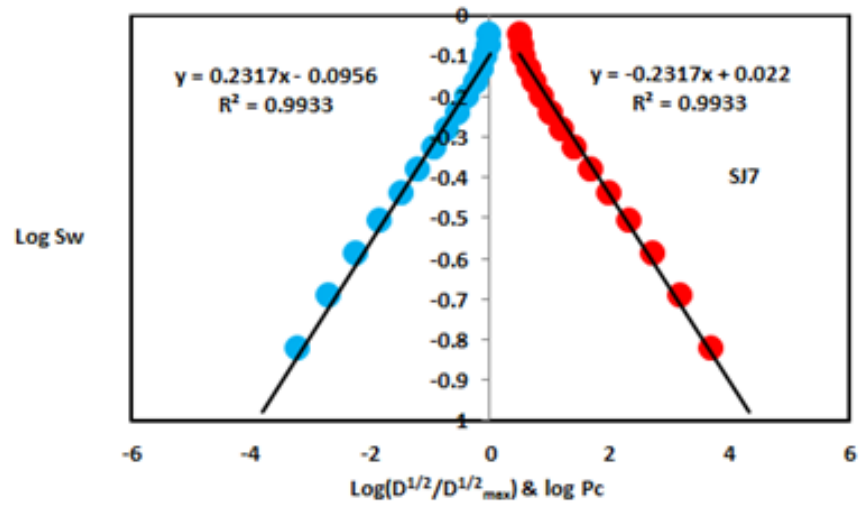

Figure $6 \log \left(D^{1 / 2} / D_{\max }^{1 / 2}\right) \& \log$ Pc versus log SW for sample SJ7.

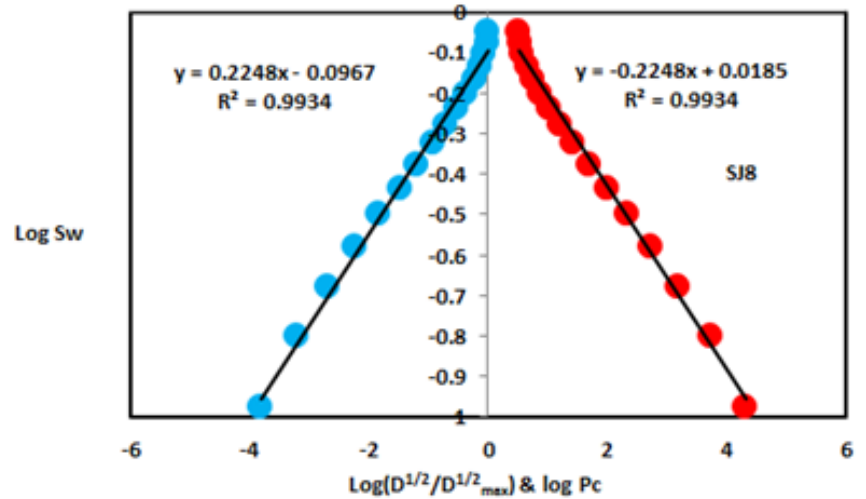

Figure $7 \mathrm{Log}\left(\mathrm{D}^{1 / 2} / \mathrm{D}_{\max }{ }^{1 / 2}\right)$ \& $\log \mathrm{Pc}$ versus log Sw for sample SJ8.

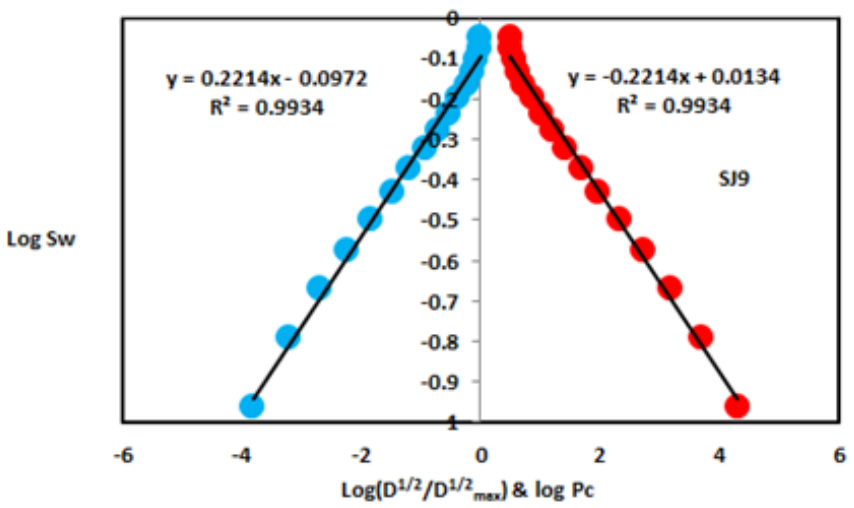

Figure 8 Log $\left(D^{1 / 2} / D_{\max }^{1 / 2}\right) \& \log$ Pc versus log Sw for sample SJ9.

On the other hand, the Upper Shajara reservoir separated from the Middle Shajara reservoir by yellow green mudstone as revealed in
Figure 1. It is defined by three samples so called SJ11, SJ12, SJ13 as explained in Table 1. Their positive slopes of the first procedure and negative slopes of the second procedure are displayed in Figures 9-11. Moreover, their diffusion coefficient fractal dimension and capillary pressure fractal dimension are also higher than those of sample SJ3 and SJ4 from the Lower Shajara Reservoir due to an increase in their permeability as clarified in Table 1 .

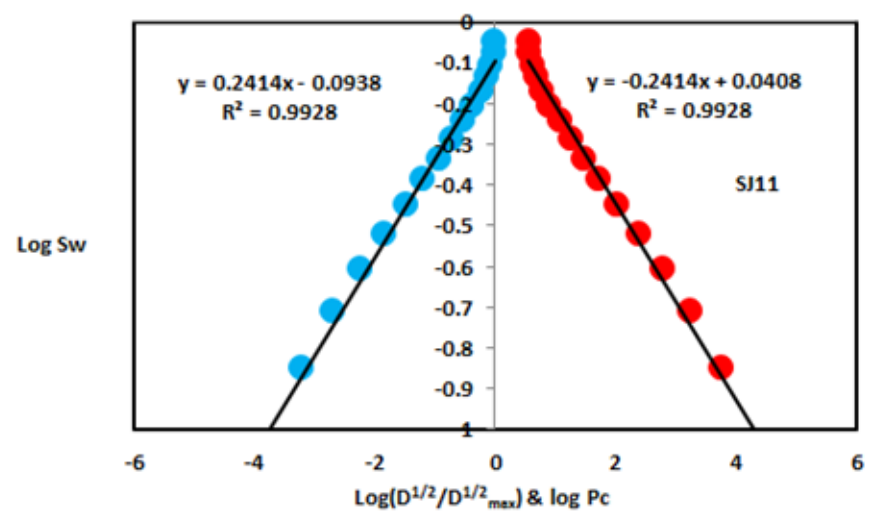

Figure $9 \log \left(D^{1 / 2} / D_{\text {max }}^{1 / 2}\right) \& \log$ Pc versus log SW for sample SJII.

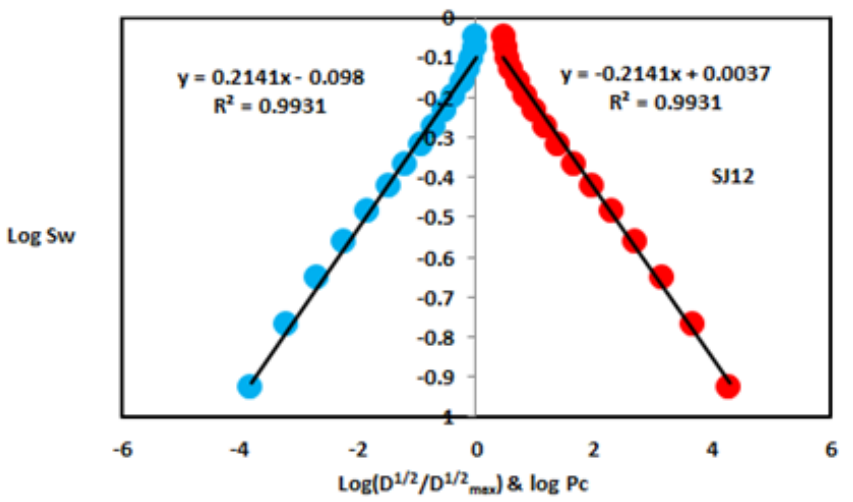

Figure 10 Log (DI/2/DmaxI/2) \& log Pc versus log Sw for sample SJI2.

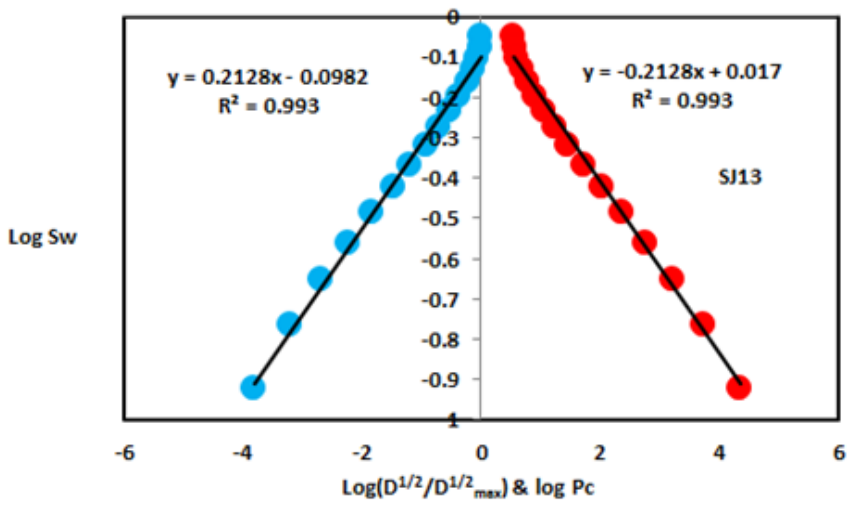

Figure II Log (DI/2/DmaxI/2) \& log Pc versus log Sw for sample SJI3.

Overall a plot of diffusion coefficient fractal dimension versus capillary pressure fractal dimension as shown in Figure 12 reveals three permeable zones of varying Petrophysical properties. Such variation in fractal dimension can account for heterogeneity which is a key parameter in reservoir quality assessment. This reservoir heterogeneity was also confirmed by plotting positive slope of the first 
procedure versus negative slope of the second procedure as described in Figure 13.

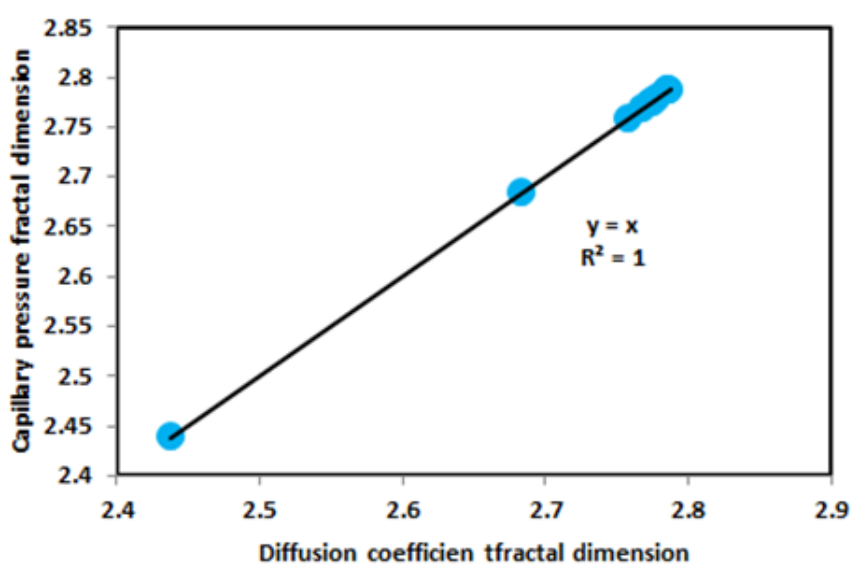

Figure I 2 Diffusion coefficient fractal dimension versus capillary pressure fractal dimension.

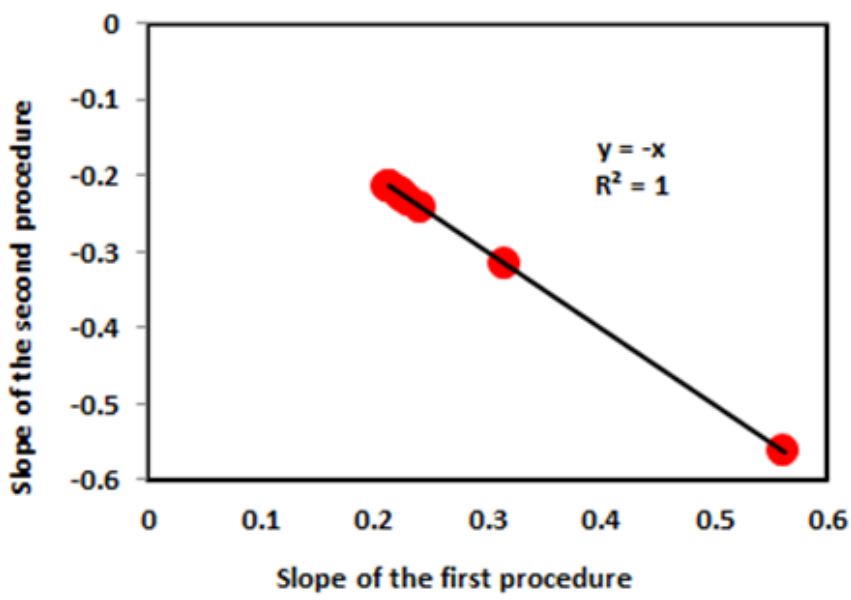

Figure I 3 Slope of the first procedure versus slope of the second procedure.

\section{Conclusion}

The sandstones of the Shajara Reservoirs of the PermoCarboniferous Shajara Formation were divided here into three units based on diffusion coefficient fractal. The Units from bottom to top are Lower Shajara Diffusion Coefficient Fractal dimension unit, Middle Shajara Diffusion Coefficient Fractal dimension unit, and Upper Shajara Diffusion Coefficient Fractal dimension unit. These units were also confirmed by capillary pressure fractal dimension. The heterogeneity increases with increasing permeability, increasing fractal dimension, decreasing compaction owing to possibility of having interconnected channels.

\section{Acknowledgments}

The author would like to thank College of Engineering, King Saud
University, Department of Petroleum and Natural Gas Engineering, Department of Chemical Engineering, Research Centre at College of Engineering and King Abdulla Institute for Research and Consulting Studies for their supports.

\section{Conflicts of interest}

The authors declared there is no conflict of interest.

\section{References}

1. Frenkel J. On the theory of seismic and seismoelectric phenomena in a moist soil. Journal of Physics. 1944;3:230-241.

2. Li K, Williams W. Determination of capillary pressure function from resistivity data. Transp Porous Media. 2007;67(1):1-15.

3. Revil A, Jardani A. Seismoelectric response of heavy oil reservoirs: theory and numerical modelling. Geophys J Int. 2010;180(2):781-797.

4. Dukhin A, Goetz P, Thommes M. Seismoelectric effect: a nonisochoric streaming current. 1 experiment. J Colloid Interface Sci. 2010;345(2):547-553.

5. Guan W, Hu J, Wang Z. Permeability inversion from low-frequency seismoelectric logs in fluid-saturated porous formations. Geophys Prospect. 2012;61(1):120-133.

6. Hu H, Guan W, Zhao W. Theoretical studies of permeability inversion from seismoelectric logs. Geophysical Research Abstracts. 2012.

7. Borde $\mathrm{C}, \mathrm{S}^{\prime}$ en'echal $\mathrm{P}$, Barri 'ere $\mathrm{J}$, et al. Impact of water saturation on seismoel+ectric transfer functions: a laboratory study of co-seismic phenomenon. Geophys J Int. 2015;200(3):1317-1335.

8. Jardani A, Revi A. Seismoelectric couplings in a poroelastic material containing two immiscible fluid phases. Geophys J Int. 2015;202(2):850 870 .

9. Holzhauer J, Brito D, Bordes C, et al. Experimental quantification of the seismoelectric transfer function and its dependence on conductivity and saturation in loose sand. Geophysics Prospect. 2016;65(4):1097-1120.

10. Rong P, Wei J-X, Di B-R, et al. Experimental research on seismoelectric effects in sandstone. Applied Geophysics. 2016;13(3):425-436.

11. Djuraev U, Jufar S R, Vasant P. Numerical Study of frequency-dependent seismoelectric coupling in partially-saturated porous media. MATEC Web of Conferences. 2017.

12. Alkhidir KEME. Pressure head fractal dimension for characterizing Shajara Reservoirs of the Shajara Formation of the Permo-Casrboniferous Unayzah Group, Saudi Arabia. Arch Pet Environ Biotech. 2017;(2):1-7.

13. Alkhidir KEME. Geometric relaxation time of induced polarization fractal dimension for characterizing Shajara Reservoirs of the Shajara Formation of the Permo-Carboniferous Unayzah Group, Saudi Arabia. Scifed J Petroleum. 2018;2(1):1-6.

14. Alkhidir KEME. Geometric relaxation time of induced polarization fractal dimensionfor characterizing Shajara Reservoirs of the Shajara formation of the Permo-Carboniferous Unayzah Group-Permo. Int J Pet Res. 2018;2(1):105-108.

15. Alkhidir KEME. Arithmetic relaxation time of induced polarization fractal dimension for characterizing Shajara Reservoirs of the Shajara Formation. Nanosci and Nanotechnol. 2018;2(1):1-8. 